\title{
Classical novae in OGLE data
}

\section{Przemek Mróz ${ }^{* \dagger}$}

Warsaw University Observatory, Warsaw, Poland

E-mail: pmrozeastrouw.edu.pl

I present the results of a systematic search for classical novae in the Galactic bulge and Magellanic Clouds in data from the OGLE survey. I measured nova rates in all those environments: $13.8 \pm$ $2.6 \mathrm{yr}^{-1}$ (Milky Way bulge), $2.4 \pm 0.8 \mathrm{yr}^{-1}$ (LMC), and $0.9 \pm 0.4 \mathrm{yr}^{-1}$ (SMC). The $K$-band luminosity-specific nova rate in the Galactic bulge is significantly higher than in other galaxies, suggesting different nova rates per unit mass in different Galactic populations. I also provide strong upper limits on the number of very frequently erupting recurrent novae, which indicates they cannot be a significant channel of SN Ia progenitors. Finally, I identified several post-nova candidates based on their photometric variability.

The Golden Age of Cataclysmic Variables and Related Objects - III

7-12 September 2015

Palermo, Italy

* Speaker.

${ }^{\dagger}$ On behalf of OGLE collaboration. 


\section{Introduction}

The Optical Gravitational Lensing Experiment (OGLE) is one of the largest sky variability surveys. The survey is currently monitoring an area of over 3500 sq. degrees: the Galactic bulge, disk, and Magellanic Clouds (for details see Udalski et al. 2015). Regular observations of those dense sky areas allow unprecedented studies of variable stars, including classical novae and other cataclysmic variables (see Fig. 1). In this paper I present the highlights from a systematic search for classical novae in the Galactic bulge (Mróz et al. 2015) and Magellanic Clouds (Mróz et al. 2016).

\section{Nova rates}

There are two methods of measuring the nova rate in the Galaxy. In the first one, novae brighter than a certain threshold magnitude are counted and their number is corrected for the completeness and selection bias. In the other approach, the Galactic nova rate is extrapolated from extragalactic nova rates (which are much easier to measure than for the Milky Way). Both methods yield a broad range of values, from 10 to $70 \mathrm{yr}^{-1}$. Moreover, the uncertainties of individual measurements are very high, from 30 to $50 \%$.

In the systematic search for nova eruptions in data from the OGLE survey, I found a large sample of objects: 39 in the Galactic bulge, 13 in the LMC, and 7 in the SMC. However, observed nova rates have to be corrected for the completeness. I will describe below how to estimate the completeness for the Galactic bulge data.

This procedure can be divided into a few steps. First, I drew a random sample of novae from a realistic spatial distribution and subsequently estimated what fraction of all of stars fall in the survey footprint. I assumed that the distribution of novae in the Galactic bulge follows the density of stars. This assumption is satisfied in neighboring galaxies, like M31 or LMC. I approximated the bulge as a triaxial ellipsoid, which is slightly inclined to the Sun - Galactic Center line. Parameters of this distribution were taken from the Besançon model (Robin et al. 2003).

In the next step, I estimated the apparent brightness in the peak for each nova from my mock catalog, to ensure it is bright enough to be detected by the survey. I chose objects brighter than $I=17$ in the peak (fainter ones could be mistaken with other erupting variables, like dwarf novae of WZ Sge type). I assumed that the luminosity function of novae is a Gaussian with a mean value of -7 mag and a dispersion of $1 \mathrm{mag}$. I also estimated the extinction toward each nova by integrating the density of the interstellar matter along the path from the observer to the star. Reddening values were then normalized to match the VVV extinction maps (Gonzalez et al. 2012).

In the third and final step, I estimated the probability of detection of each nova based on the history of observations of each field. I assumed that eruptions are distributed uniformly in time and each one can be detected for 20 days after the start of the outburst. This leads to the overall completeness of $16.7 \%$ (for the years 2001-2013). For OGLE-IV data (2010-2013), the efficiency of detection is much higher: $36.1 \%$. After correcting observed nova rates for the completeness, I obtained the true Galactic bulge nova rate of $13.8 \pm 2.6 \mathrm{yr}^{-1}$. 

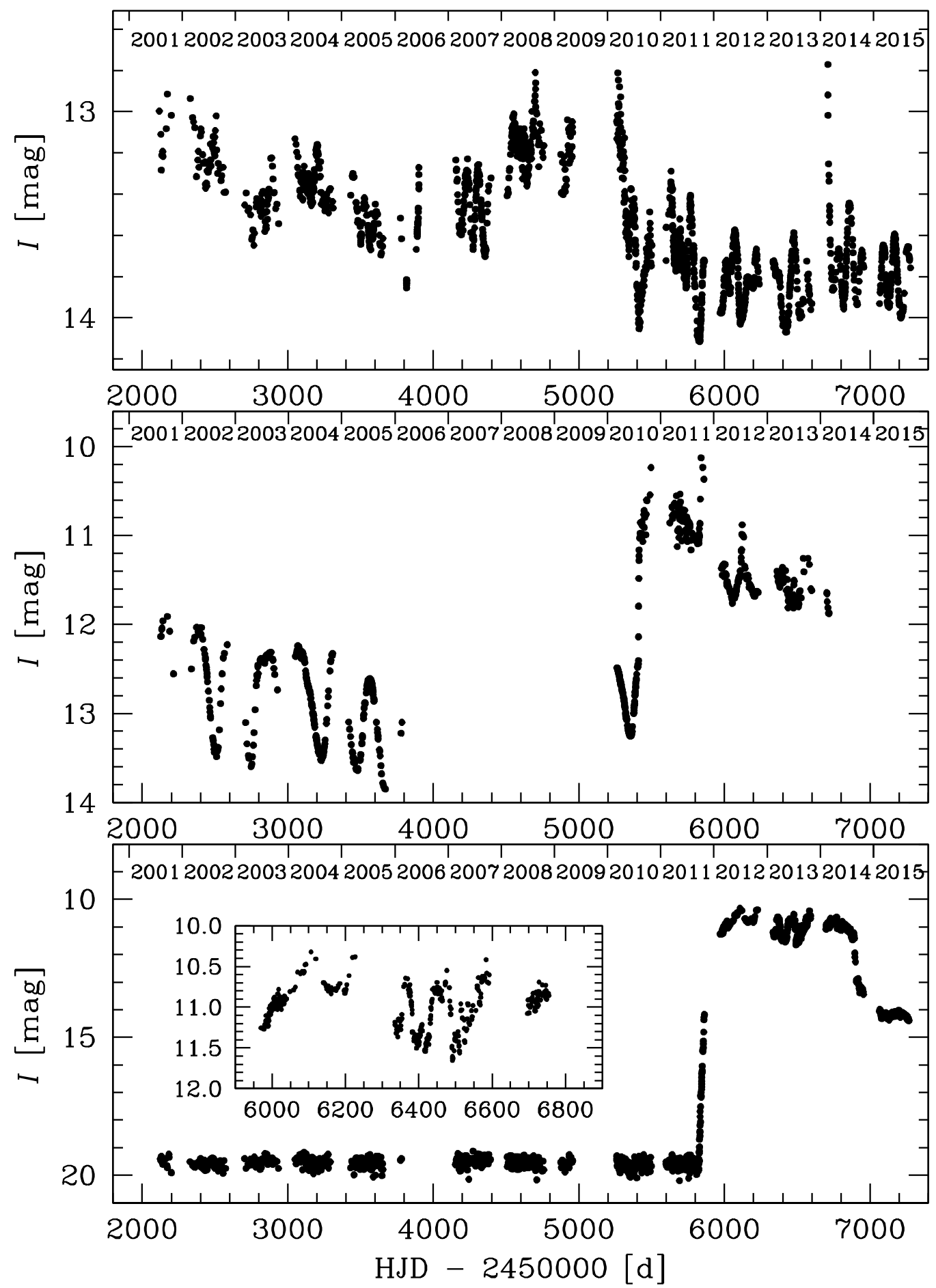

Figure 1: Light curve gallery. From top to bottom: 1) Quiescent light curve of recurrent nova V745 Sco is dominated by semiregular pulsations of the red giant secondary. There are two periods of 136.5 and $77.4 \mathrm{~d}$, which likely correspond to the fundamendal-mode and first overtone radial pulsations of the red giant. 2) The progenitor of symbiotic nova V5590 Sgr was a Mira star with pulsation period of $236 \mathrm{~d}$. 3) The very long outburst of OGLE-2011-BLG-1444: a slow classical nova or symbiotic nova. 
In an analogous way, I estimated the completeness of the sample of the Magellanic Clouds novae. This allowed me to measure nova rates in the Large $\left(2.4 \pm 0.8 \mathrm{yr}^{-1}\right)$ and Small Magellanic Cloud $\left(0.9 \pm 0.4 \mathrm{yr}^{-1}\right)$. The LMC nova rate is in agreement with previous estimates, while the SMC rate is much higher than previously thought (five out of seven SMC novae used in my calculations were previously unknown).

I estimated luminosity-specific nova rates $^{1}$ (LSNRs) for each environment (Galactic bulge, LMC, SMC). For the majority of galaxies with measured nova rates, the LSNR seem to be roughly constant: $(1-3) \mathrm{yr}^{-1}\left(10^{10} L_{\odot, K}\right)^{-1}$ (see Fig. 2). However, for the Magellanic Clouds I measured LSNR of $6.5 \pm 2.2 \mathrm{yr}^{-1}\left(10^{10} L_{\odot, K}\right)^{-1}$ in the LMC and $7.5 \pm 3.1 \mathrm{yr}^{-1}\left(10^{10} L_{\odot, K}\right)^{-1}$ in the SMC. This agrees with some of previous measurements (e.g. Della Valle et al. 1994), but the largest source of systematic uncertainty is a poorly known brightness of the Clouds (Williams \& Shafter 2004). High LSNRs can be explained by the star formation history in the Magellanic Clouds, specifically a re-ignition of the star formation rate a few Gyr ago (Mróz et al. 2016).

Assuming the $K$-band brightness of the Galactic bulge of $(1.6 \pm 0.4) \cdot 10^{10} L_{\odot, K}$ (Drimmel \& Spergel 2001), I measured the LSNR of $8.6 \pm 2.7 \mathrm{yr}^{-1}\left(10^{10} L_{\odot, K}\right)^{-1}$. The cause of high LSNR in the Galactic bulge remains unknown, but it indicates the presence of two populations of Galactic novae, characterized by different nova rates per unit brightness/mass (Della Valle et al. 1992, Della Valle \& Livio 1998).

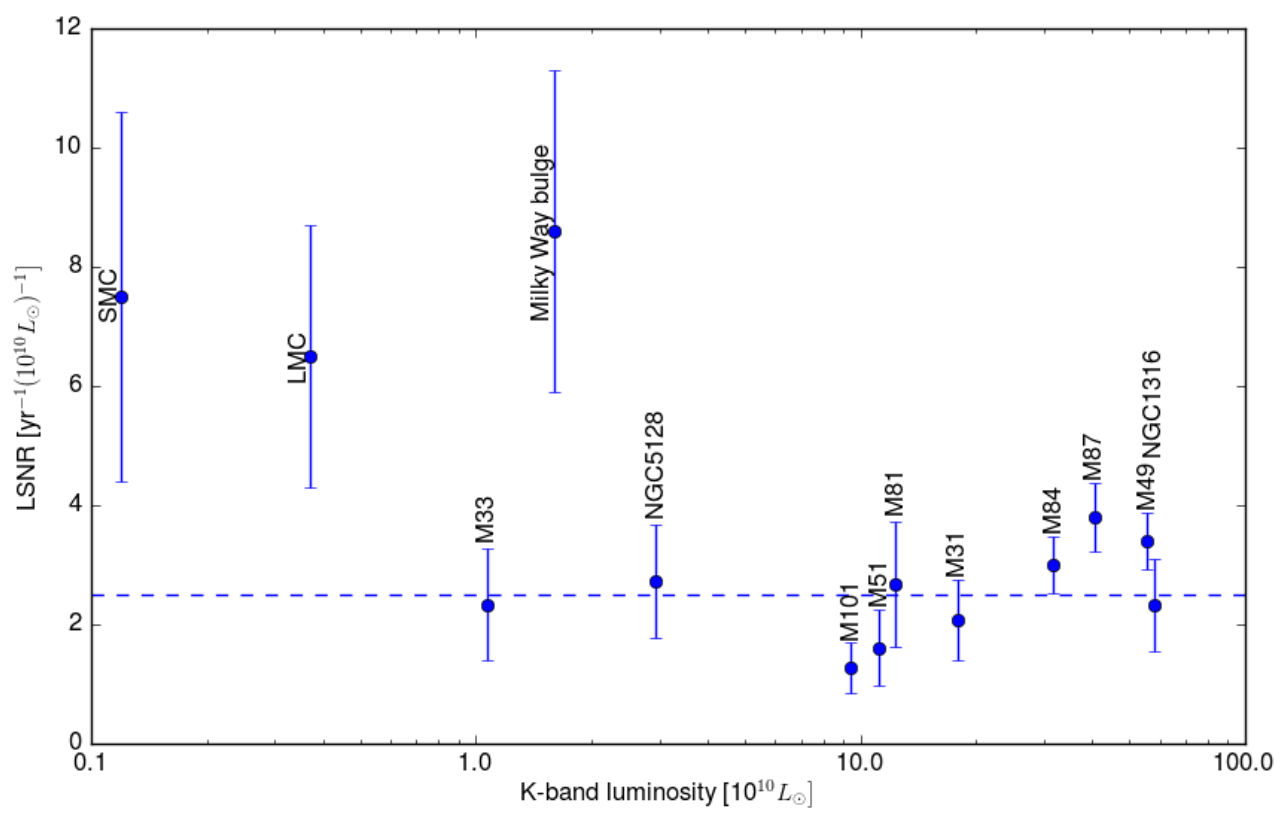

Figure 2: LSNRs in different galaxies. A dashed line corresponds to the typical LSNR of $2.5 \mathrm{yr}^{-1}$ $\left(10^{10} L_{\odot, K}\right)^{-1}$ found for many neighboring galaxies. The LSNR in the Milky Way bulge is significantly higher, suggesting different nova rates per unit mass in different Galactic populations. Data are taken from Williams \& Shafter (2004), Curtin et al. (2015), and this work.

\footnotetext{
${ }^{1}$ LSNR is a nova rate divided by the $K$-band luminosity of a parent galaxy.
} 


\section{Frequently erupting recurrent novae}

The cadence of OGLE observations varies from 20 min (in the central Galactic bulge fields) to once per 2-4 days. Hence, the survey is very sensitive to frequently erupting recurrent novae. For example, we should discover at least two eruptions for $40 \%$ of all novae with recurrence time of $300 \mathrm{~d}$ in the Galactic bulge. Our sensitivity for recurrence timescales of $300 \mathrm{~d}, 1000 \mathrm{~d}$, and $5 \mathrm{yr}$ for three different environments (Galactic bulge, LMC, SMC) is shown in Table 1.

Since our sensitivity is very high, and the number of known frequently erupting novae is zero, we can set very strong upper limits on the number of such objects in the Galactic bulge and Magellanic Clouds. This leads to the conclusion that the death rate of such frequently erupting recurrent novae is smaller than $1 \%$ of the Type Ia supernova rate in the Milky Way, improving previous estimates by a factor of a few (Della Valle \& Livio 1996). We also did not find any faint and very fast novae (Kasliwal et al. 2009).

\begin{tabular}{lrrr}
\hline$\tau_{\text {rec }}$ & Milky Way bulge & LMC & SMC \\
\hline $300 \mathrm{~d}$ & $40 \%$ & $76 \%$ & $87 \%$ \\
$1000 \mathrm{~d}$ & $14 \%$ & $59 \%$ & $75 \%$ \\
$5 \mathrm{yr}$ & $7.0 \%$ & $16 \%$ & $28 \%$ \\
\hline
\end{tabular}

Table 1: A probability of detection of at least two eruptions of a RN with a recurrence timescale $\tau_{\text {rec }}$.

\section{Post-novae}

Our knowledge of what happens in the binary after the nova eruption is very limited, partially because of the lack of observational data. For example, only a small fraction of pre-1980 novae has been identified in quiescence. I used long-term, high-quality OGLE observations to identify post-novae showing different types of photometric variability. I searched for periodic variations (eclipses or ellipsoidal modulations) as well as long-term variability (e.g., pulsations of the evolved secondaries). I also searched for any kind of suspicious light curve behavior, like sudden brightenings (dwarf nova outbursts / recurrent nova eruptions) or fading events.

I analyzed light curves of all objects from OGLE databases which lie within $3^{\prime \prime}$ around the positions of over 80 known old novae. I found nine likely post-novae, which show variability on an orbital period as well as a few with semi-regular photometric variations. All candidates are listed in Mróz et al. (2015).

\section{Prospects for the future}

The OGLE survey is observing the Galactic disk in the course of the OGLE-IV Galaxy Variability Survey. The disk data should reveal at least several classical novae. I should be able to estimate the Galactic disk nova rate and hence the total nova rate in the Milky Way. Observations of the Galactic disk novae should also provide the final answer to the question if there exist two populations of Galactic novae. 


\section{Acknowledgments}

P.M. is supported by the "Diamond Grant" No. DI2013/014743 funded by the Polish Ministry of Science and Higher Education. The OGLE project has received funding from the National Science Center, Poland, grant MAESTRO 2014/14/A/ST9/00121 to Andrzej Udalski.

\section{References}

[1] C. Curtin, A. W. Shafter, C. J. Pritchet, et al., Exploring the Role of Globular Cluster Specific Frequency on the Nova Rates in Three Virgo Elliptical Galaxies, ApJ, 811, 34 (2015)

[2] M. Della Valle, A. Bianchini, M. Livio, and M. Orio, On the possible existence of two classes of progenitors for classical novae, A\&A, 266, 232 (1992)

[3] M. Della Valle \& M. Livio, On the Frequency of Occurrence of Recurrent Novae and Their Role as Type IA Supernova Progenitors, ApJ, 473, 240 (1996).

[4] M. Della Valle \& M. Livio, The Spectroscopic Differences between Disk and Thick-Disk/Bulge Novae, ApJ, 506, 818 (1998)

[5] M. Della Valle, L. Rosino, A. Bianchini, and M. Livio, The nova rate in galaxies of different Hubble types, A\&A, 287, 403 (1994)

[6] R. Drimmel and D. N. Spergel, Three-dimensional structure of the Milky Way disk: the distribution of stars and dust beyond $0.35 R_{\odot}, \mathrm{ApJ}, 556,181$ (2001)

[7] O. A. Gonzalez, M. Rejkuba, M. Zoccali, et al., Reddening and metallicity maps of the Milky Way bulge from VVV and 2MASS. II. The complete high resolution extinction map and implications for Galactic bulge studies, A\&A, 543, A13 (2012)

[8] M. M. Kasliwal, S. B. Cenko, S. R. Kulkarni, et al., Discovery of a New Photometric Sub-class of Faint and Fast Classical Novae, ApJ, 735, 94 (2011)

[9] D. Maoz, F. Mannucci, and G. Nelemans, Observational Clues to the Progenitors of Type Ia Supernovae, ARA\&A, 52, 107 (2014)

[10] P. Mróz, R. Poleski, A. Udalski, et al., Recurrent and symbiotic novae in data from the Optical Gravitational Lensing Experiment, MNRAS, 443, 784 (2014)

[11] P. Mróz, A. Udalski, R. Poleski, et al., OGLE Atlas of Classical Novae. I. Galactic Bulge Objects, ApJS, 219, 26 (2015)

[12] P. Mróz, A. Udalski, R. Poleski, et al., OGLE Atlas of Classical Novae. II. Magellanic Clouds, ApJS, 222, 9 (2016)

[13] A. C. Robin, C. Reylé, S. Derrière, and S. Picaud, A synthetic view on structure and evolution of the Milky Way, A\&A, 409, 523 (2003)

[14] A. Udalski, M. K. Szymański, and G. Szymański, OGLE-IV: Fourth Phase of the Optical Gravitational Lensing Experiment, Acta Astron., 65, 1 (2015)

[15] S. J. Williams and A. W. Shafter, On the Nova Rate in M33, ApJ, 612, 867 (2004) 


\section{DISCUSSION}

MARTIN HENZE: For your simulation, did you use a sample of CN light curves or a control time for detection? How good is your cadence?

PRZEMEK MRÓZ: The typical cadence in the Galactic bulge fields varies from 20 min to one observation per 2-3 nights. In simulations, I used fixed length of "detectability window". For example, in the estimation of sensitivity for recurrent novae I tried two values of five and ten days and in both cases our sensitivity is very high.

JOANNA MIKOLAJEWSKA: How do you estimate the SN Ia rate for the Galactic bulge to compare with the $\mathrm{RNe}$ death rate?

PRZEMEK MRÓZ: I compared the RNe death rate to the overall SN Ia rate in the Milky Way of one per $140 \pm 50 \mathrm{yr}$ from the latest review paper by Maoz et al. (2014). However, since Type Ia $\mathrm{SNe}$ are observed in all galactic environments and the Galactic bulge contains roughly $20 \%$ of the Milky Way light, the Type Ia SN rate in the Galactic bulge should be roughly one per 500 - 1000 yr. Since my upper limit on the number of frequently erupting recurrent novae in the Galactic bulge is very strong, this does not affect my conclusion that such recurrent novae cannot be a significant channel of Type Ia SN progenitors. 